\title{
Generation of Vertical Fine Structure by the Internal Waves with the Regard for Turbulent Viscosity and Diffusion
}

\author{
A. A. Slepyshev*, A. V. Nosova \\ Marine Hydrophysical Institute of RAS, Sevastopol, Russian Federation \\ *slep55@mail.ru
}

Purpose. The aim is to study the mechanism of formation of a vertical fine structure due to the mass vertical transfer by the internal waves taking into account turbulent viscosity and diffusion as well as to investigate influence of the critical layers on the dispersion curves of internal waves.

Methods and Results. In the Boussinesq approximation, the free inertia-gravity internal waves in a vertically inhomogeneous flow are considered with the regard for the horizontal turbulent viscosity and diffusion. The equation for the amplitude of vertical velocity of the internal waves contains a small parameter (in the dimensionless variables) proportional to the value of the horizontal turbulent viscosity. The solution of this equation is realized in a form of the asymptotic series of this parameter. In the zero approximation, the second-order homogeneous boundary value problem determined the vertical structure mode is solved numerically by the implicit third-order accuracy Adams scheme for real profiles of the Brent-Väisälä frequency and the current velocity. At the fixed wave frequency, the wave number is determined by the shooting method. In the first order with respect to the indicated parameter, the semi-homogeneous boundary value problem is also solved numerically according to the implicit Adams scheme of the third order of accuracy. A unique solution is found which is orthogonal to the solution of the corresponding homogeneous boundary value problem. The condition of this boundary value problem solvability yields the wave attenuation decrement. The dispersion curves of the first two modes are cut off in the lowfrequency region (the second mode is at a higher frequency), that is due to influence of the critical layers, where the wave frequency with the Doppler shift is inertial. It is shown that the mass vertical wave flux differs from zero and leads to correction (not oscillating on the wave time scale) of the average density, i. e. the internal wave generate fine structure that is of an irreversible character.

Conclusions. When the horizontal turbulent viscosity and diffusion are taken into consideration, the mass vertical wave flux differs from zero and leads to generation of the vertical fine structure. The mass wave flux exceeds the turbulent one. The vertical scales of the generated vertical fine structure correspond to the actually observed ones.

Keywords: internal waves, wave flux of mass, fine structure, critical layers.

Acknowledgments: the investigation was carried out within the framework of the state task on theme No. 0827-2018-0003 "Fundamental studies of oceanic processes determined state and evolution of marine environment being affected by natural and anthropogenic factors, based on observation and modeling methods".

For citation: Slepyshev, A.A. and Nosova, A.V., 2020. Generation of Vertical Fine Structure by the Internal Waves with the Regard for Turbulent Viscosity and Diffusion. Physical Oceanography, [e-journal] 27(1), pp. 3-17. doi:10.22449/1573-160X-2020-1-3-17

DOI: $10.22449 / 1573-160 X-2020-1-3-17$

(C) A.A. Slepyshev, A.V. Nosova, 2020

(C) Physical Oceanography, 2020

The vertical fine structure of hydrophysical fields was discovered in the second half of the twentieth century after the creation and further improvement of high-resolution sounding equipment [1-4]. It was found out that 
the temperature and salinity vertical profiles are very indented vertically (without density inversions) and constitute a "layer cake" ${ }^{1}$. The cause for this layering was not clear.

According to one concept, small-scale turbulence is generated due to KelvinHelmholtz local hydrodynamic instability and breaking of internal waves [5-16]. According to other concepts, the double diffusion mechanism leads to the formation of stepped structures in the ocean $[17,18]$. The role of intrusive separation in the areas of fronts and synoptic eddies [19-21] should be noted. Hydrodynamic instability and intrusion separation often work together, creating a small-scale layering - a microstructure in the ocean [3]. The wave mechanism for generating a vertical fine structure due to nonlinear effects during the propagation of internal wave trains (without breaking) also merits attention. Its essence is that during the propagation of weakly nonlinear internal wave train, the scale-average current waves and non-oscillating correction to the density are generated. This correction is interpreted as a wave-generated fine structure [22, 23]. The mentioned correction to the density is proportional to the square of wave amplitude, and after the wave train passage the unperturbed stratification profile regains. Thus, the fine structure generated by the wave train is reversible.

With regard to turbulent viscosity and diffusion, the internal waves attenuate $[24,25]$. The vertical wave fluxes of heat, salt and momentum are nonzero [26, 27]. Below it will be shown that the wave flux of mass $\overline{\rho w}$ ( $\rho, w$ are wave perturbations of density and vertical velocity, respectively) is nonzero and leads to average density profile deformation - to a fine structure that is generated by the wave and has an irreversible character. Since the coefficients of horizontal turbulent viscosity and diffusion are three to four orders of magnitude greater than the corresponding coefficients of vertical turbulent viscosity and diffusion, the latter are neglected.

Statement of the problem. Free internal waves in a basin of constant depth are considered taking into account the Earth rotation in the presence of an average plane-parallel current with a vertical velocity shift. The coefficients of horizontal turbulent viscosity and diffusion are assumed to be constant. The amplitude of the vertical velocity, dispersion relation and wave attenuation decrement are found in the linear approximation. In the second order of the wave amplitude the wave flux of mass and non-oscillating on the wave scale density correction are determined. This density correction is a wave-generated fine structure. We introduce dimensionless variables [27] (a prime symbol marks the dimensional physical quantities):

$$
\begin{aligned}
& x^{\prime}=H x, \quad y^{\prime}=H y, \quad z^{\prime}=H z, \quad t^{\prime}=t / \omega_{*}, \\
& u^{\prime}=H \omega_{*} u, \quad v^{\prime}=H \omega_{*} v, \quad w^{\prime}=H \omega_{*} w, \quad U_{0}^{\prime}=H \omega_{*} U_{0},
\end{aligned}
$$

\footnotetext{
Panteleev, N.A., Okhotnikov, I.N. and Slepyshev, A.A., 1993. Small-Scale Structure and Dynamics of the Ocean. Kiev: Naukova Dumka, 193 p. (in Russian); Fedorov, K.N., 1976. Fine Thermohaline Structure of Oceanic Waters. Leningrad: Gidrometeoizdat, 184 p. (in Russian).
} 


$$
\begin{aligned}
& \rho^{\prime}=\rho_{0}^{\prime}(0) H \omega_{*}^{2} \rho / g, \quad \rho_{0}^{\prime}(z)=\rho_{0}^{\prime}(0) H \omega_{*}^{2} \rho_{0}(z) / g, \\
& P^{\prime}=\rho_{0}^{\prime}(0) H^{2} \omega_{*}^{2} P, \quad K^{\prime}=K \mu, \quad M^{\prime}=M \mu, \quad f^{\prime}=f \omega_{*},
\end{aligned}
$$

where $x, y, z$ are two horizontal and a vertical coordinate, $z$ axis is directed upwards; $\omega_{*}$ is a characteristic wave frequency; $u, v, w$ are two horizontal and a vertical component of wave current velocity, respectively; $\rho$ and $P$ are wave perturbations of density and pressure; $\rho_{0}$ is non-perturbed mean water density; $H$ is a sea depth; $K, M$ are coefficients of horizontal turbulent viscosity and diffusion; $\mu$ is a characteristic value of horizontal turbulent viscosity; $U_{0}(z)$ mean flow velocity; $f$ is the Coriolis parameter.

A system of hydrodynamics equations for wave perturbations in the Boussinesq approximation has the following form:

$$
\begin{gathered}
\frac{D u}{D t}-f v+w \frac{d U_{0}}{d z}=-\frac{\partial P}{\partial x}+\varepsilon K \Delta_{h} u \\
\frac{D v}{D t}+f u=-\frac{\partial P}{\partial y}+\varepsilon K \Delta_{h} v \\
\frac{D w}{D t}=-\frac{\partial P}{\partial z}+\varepsilon K \Delta_{h} w-\rho \\
\frac{D \rho}{D t}=-w \frac{d \rho_{0}}{\partial z}+\varepsilon M \Delta_{h} \rho \\
\frac{\partial u}{\partial x}+\frac{\partial v}{\partial y}+\frac{\partial w}{\partial z}=0
\end{gathered}
$$

Here $\varepsilon=\mu / \omega_{*} H^{2}$ is a small parameter proportional to the horizontal turbulent viscosity value; $\Delta_{h}$ is horizontal Laplace operator, $\Delta_{h}=\frac{\partial^{2}}{\partial x^{2}}+\frac{\partial^{2}}{\partial y^{2}}$; the action of $D / D t$ operator is revealed by the formula

$$
\frac{D}{D t}=\frac{\partial}{\partial t}+\left(u+U_{0}\right) \frac{\partial}{\partial x}+v \frac{\partial}{\partial y}+w \frac{\partial}{\partial z}
$$


Boundary conditions at the sea surface $(z=0)$ are a rigid lid condition that filters internal waves from the surface ones ${ }^{2}$ and the absence of tangential stresses [25]:

$$
\begin{aligned}
w(0) & =0, \\
K \frac{\partial w}{\partial x}=0, \quad K \frac{\partial w}{\partial y} & =0, \quad z=0 .
\end{aligned}
$$

Boundary conditions at the bottom are the impermeability condition and the absence of tangential stresses (smooth slip condition [25]):

$$
w=0, \quad K \frac{\partial w}{\partial x}=0, \quad K \frac{\partial w}{\partial y}=0, \quad z=-1 .
$$

The tangential stresses at the bottom are zero, since the vertical exchange coefficients are neglected.

Linear approximation. The solutions of linear approximations we seek in the form

$$
\begin{gathered}
u_{1}=u_{10}(z) A \exp (i \theta)+c . c ., \quad v_{1}=v_{10}(z) A \exp (i \theta)+c . c ., \quad w_{1}=w_{10}(z) A \exp (i \theta)+c . c ., \\
P_{1}=P_{10}(z) A \exp (i \theta)+c . c ., \quad \rho_{1}=\rho_{10}(z) A \exp (i \theta)+c . c .
\end{gathered}
$$

where $c$. $c$. are complex conjugate terms; $A$ is the an amplitude factor; $\theta$ is a wave phase; $\partial \theta / \partial x=k, \partial \theta / \partial t=-\omega, k$ is a horizontal wave number, $\omega$ is a wave frequency. It is assumed that the wave propagates along $x$ axis.

After substituting (8) into system (1)-(5), the relationship of the amplitude functions follows $u_{10}, v_{10}, \rho_{10}, P_{10}$ with $w_{10}$ :

$$
\begin{gathered}
u_{10}=\frac{i}{k} \frac{d w_{10}}{d z}, \quad \Omega=\omega-k \cdot U_{0}, \\
P_{10}=\frac{i}{k}\left[\frac{\Omega}{k} \frac{d w_{10}}{d z}+\frac{d U_{0}}{d z} w_{10}-\frac{i f^{2}}{k\left(i \Omega-\varepsilon k^{2} K\right)} \frac{d w_{10}}{d z}+i \varepsilon k K \frac{d w_{10}}{d z}\right], \\
\rho_{10}=\frac{w_{10}}{i \Omega-\varepsilon k^{2} M} \frac{d \rho_{0}}{d z}, \quad v_{10}=\frac{i f}{k\left(i \Omega-\varepsilon k^{2} K\right)} \frac{d w_{10}}{d z} .
\end{gathered}
$$

Function $w_{10}$ satisfies the equation

$$
\begin{aligned}
& \left(\Omega+i \varepsilon k^{2} K\right)\left[\Omega^{2}+2 i \Omega k^{2} K \varepsilon-f^{2}-\varepsilon^{2} k^{4} K^{2}\right] \frac{d^{2} w_{10}}{d z^{2}}-k f^{2} \frac{d U_{0}}{d z} \frac{d w_{10}}{d z}+ \\
& +k\left[\left(\frac{d^{2} U_{0}}{d z^{2}}-k \Omega-i \varepsilon k^{3} K\right)\left(\Omega+i \varepsilon k^{2} K\right)^{2}+k N^{2} \frac{\left(\Omega+i \varepsilon k^{2} K\right)^{2}}{\left(\Omega+i \varepsilon k^{2} M\right)}\right] w_{10}=0,
\end{aligned}
$$

\footnotetext{
${ }^{2}$ Miropolsky, Yu.Z., 1981. Dynamics of Internal Gravitational Waves in the Ocean. Leningrad: Gidrometeoizdat, p. 30. (in Russian).
} 
where $N^{2}=-d \rho_{0} / d z$ is a square of Brunt - Väisälä frequency.

Boundary conditions for $w_{10}$ :

$$
\text { at } z=0,-1 \quad w_{10}=0 .
$$

Boundary conditions (6), (7) are satisfied automatically. Equation (11) has a small parameter $\varepsilon$. Following the method described in [28], the solution $w_{10}$ and frequency $\omega$ are represented in the form of asymptotic series by $\varepsilon$ parameter:

$$
\begin{gathered}
w_{10}(z, \varepsilon)=w_{0}(z)+\varepsilon w_{1}(z)+\ldots, \\
\omega=\omega_{0}+\varepsilon \omega_{1}+\ldots .
\end{gathered}
$$

After substituting expansions (13), (14) into (11), (12) we obtain the boundaryvalue problem for $w_{0}$ in the zero approximation by $\varepsilon$ :

$$
L w_{0}=\frac{d^{2} w_{0}}{d z^{2}}-\frac{k f^{2}}{\Omega_{0}\left(\Omega_{0}^{2}-f^{2}\right)} \frac{d U_{0}}{d z} \frac{d w_{0}}{d z}+\left[\frac{d^{2} U_{0}}{d z^{2}} \Omega_{0}+k\left(N^{2}-\Omega_{0}^{2}\right)\right] \frac{k w_{0}}{\left(\Omega_{0}^{2}-f^{2}\right)}=0,
$$

where $L$ is linear differential operator; $\Omega_{0}=\omega_{0}-k \cdot U_{0}$ is a wave frequency with Doppler shift.

Boundary conditions for $w_{0}$ are as follows:

$$
w_{0}(0)=0, \quad w_{0}(-1)=0
$$

The boundary-value problem (15), (16) in the absence of a flow at $U_{0}=0$ has a countable set of eigenfunctions - a set of modes. Moreover, for each value of the wave number corresponds a certain frequency value $\omega_{0}$, satisfying the inequality $f<\omega_{0}<\max (N)$ and corresponding to the given mode. At $U_{0} \neq 0$ a discrete spectrum of real eigenfrequencies may not exist [29]. This is due to the singularities in equation (15), when $\Omega_{0}=0$ and $\Omega_{0}= \pm f$ (hydrodynamically stable flows are considered). In the presence of $\Omega_{0}=0$ singularity, there is a critical layer where the phase velocity of the wave is equal to the flow velocity $[30,31]$. When Earth's rotation is taken into account, the mentioned singularity shifts to the level where $\Omega_{0}=f$ [32]. The effect of this singularity on the dispersion curves is illustrated by the calculations below.

We introduce the notation:

$$
a(z)=-\frac{f^{2} k}{\Omega_{0}\left(\Omega_{0}^{2}-f^{2}\right)} \frac{d U_{0}}{d z}, b(z)=\frac{k}{\left(\Omega_{0}^{2}-f^{2}\right)}\left[k\left(N^{2}-\Omega_{0}^{2}\right)+\Omega_{0} \frac{d^{2} U_{0}}{d z^{2}}\right] .
$$

Then equation (15) can be written as

$$
\frac{d^{2} w_{0}}{d z^{2}}+a(z) \frac{d w_{0}}{d z}+b(z) w_{0}=0
$$


Equation (17) is reduced to a self-adjoint form by multiplying both sides of the equation by $p(z)=\exp \left(\int a(z) d z\right)$ function:

$$
L_{s} w_{0}=\frac{d}{d z}\left(p(z) \frac{d w_{0}}{d z}\right)-q(z) w_{0}=0,
$$

where $q(z)=-b(z) p(z) ; L_{s}$ is a self-adjoint differential operator.

The following approximation $w_{1}$ in the expansion (13) with respect to the parameter $\varepsilon$ satisfies the equation

$$
\begin{gathered}
L w_{1}=F_{1}(z), \\
F_{1}(z)=G\left\{\left(\omega_{1}+i k^{2} K\right)\left[k\left(k\left(3 \Omega_{0}^{2}-2 N^{2}\right)-2 \Omega_{0} \frac{d^{2} U_{0}}{d z^{2}}\right) w_{0}-\left(3 \Omega_{0}^{2}-f^{2}\right) \frac{d^{2} w_{0}}{d z^{2}}\right]+\right. \\
\left.+k \Omega_{0}\left(\omega_{1}+i k^{2} M\right)\left(k \Omega_{0}-\frac{d^{2} U_{0}}{d z^{2}}\right) w_{0}\right\}, \quad G=\frac{1}{\Omega_{0}\left(\Omega_{0}^{2}-f^{2}\right)},
\end{gathered}
$$

where $G$ is an auxiliary function.

The left side of equation (19) is reduced to a self-adjoint form by multiplying both parts of equation (19) by function $p(z)$ :

$$
L_{s} w_{1}=\Phi_{1}(z)
$$

where $\Phi_{1}(z)=p(z) F_{1}(z)$.

Boundary conditions for function $w_{1}$ :

$$
w_{1}(0)=0, \quad w_{1}(-1)=0 .
$$

The solvability condition for the boundary value problem ${ }^{3}(20),(21)$ :

$$
\int_{-1}^{0} \Phi_{1} w_{0} d z=0
$$

From here we find the expression for $\omega_{1}$ :

$$
\omega_{1}=i \frac{c}{d}
$$

${ }^{3}$ Kamke, E., 1959. Differentialgleichungen: Lösungsmethoden und Lösungen. Leipzig: Geest \& Portig K.-G. (in German). 


$$
\begin{gathered}
c=\int_{-1}^{0}\left\{\begin{array}{l}
\left.K\left[\frac{d^{2} w_{0}}{d z^{2}}\left(3 \Omega_{0}^{2}-f^{2}\right)+k w_{0}\left(2 \Omega_{0} \frac{d^{2} U_{0}}{d z^{2}}+\left(2 N^{2}-3 \Omega_{0}^{2}\right) k\right)\right]+\right] k \Omega_{0}\left(\frac{d^{2} U_{0}}{d z^{2}}-k \Omega_{0}\right) \\
+k M w_{0} G d z
\end{array}\right] \\
d=\int_{-1}^{0}\left[\frac{d^{2} w_{0}}{d z^{2}}\left(f^{2}-3 \Omega_{0}^{2}\right)+k w_{0}\left(4 k \Omega_{0}^{2}-3 \Omega_{0} \frac{d^{2} U_{0}}{d z^{2}}-2 k N^{2}\right)\right] p w_{0} G d z .
\end{gathered}
$$

Wave mass transport. The vertical wave flux of mass is determined by the formula

$$
\overline{\rho w}=\frac{w_{10} w_{10}^{*}\left|A_{1}\right|^{2}}{i \Omega-\varepsilon k^{2} M} \frac{d \rho_{0}}{d z}+c . c .,
$$

where $A_{1}=A \exp (\delta \omega \cdot t), \delta \omega=\omega_{1} / i$ is a wave attenuation decrement, $\omega_{1}$ is purely imaginary value; the bar above means averaging over the wave period. The vertical wave flux of mass leads to irreversible deformation of the density field, which can be considered as a wave-generated vertical fine structure. The equation for nonoscillating at the wave time scale correction to the mean density $\bar{\rho}$ has the following form:

$$
\frac{\partial \bar{\rho}}{\partial t}+U_{0} \frac{\partial \bar{\rho}}{\partial x}+V_{0} \frac{\partial \bar{\rho}}{\partial y}+\frac{\partial \overline{\rho u}}{\partial x}+\frac{\partial \overline{\rho v}}{\partial y}+\frac{\partial \overline{\rho w}}{\partial z}=0
$$

In the horizontally homogeneous case this equation is transformed to

$$
\frac{\partial \bar{\rho}}{\partial t}+\frac{\partial \overline{\rho w}}{\partial z}=0
$$

We integrate equation (25) over the time:

$$
\Delta \bar{\rho}=-\int_{0}^{t}\left(\frac{\partial \overline{\rho w}}{\partial z}\right) d t^{\prime}
$$

Substituting vertical wave flux of mass $\overline{\rho w}$ (24) into integral (26), after integration we obtain

$$
\overline{\Delta \rho}=\frac{1}{2 \delta \omega} \frac{\partial \overline{\rho w}^{0}}{\partial z} \cdot\left(1-\mathrm{e}^{2 \delta \omega \cdot t}\right)
$$


where $\overline{\rho w}^{0}=\frac{w_{10} w_{10}^{*}|A|^{2}}{i \Omega-\varepsilon k^{2} M} \frac{d \rho_{0}}{d z}+c . c ., A=A_{1}$ at $t=0$.

In expression (27), turning to the limit at $t \rightarrow \infty$ with regard to the fact that $\delta \omega<0$, we find $\overline{\Delta \rho}$ :

$$
\overline{\Delta \rho}=\frac{\partial \overline{\rho w}^{0}}{\partial z} \cdot \frac{1}{2 \delta \omega}
$$

The value $\overline{\Delta \rho}$, that depends on the vertical coordinate, is non-oscillating at the wave time scale correction to the average density - vertical fine structure generated by the wave. In [22, 23], a non-oscillating correction to the density, proportional to the square of the wave amplitude, was determined. After the wave train passage, the unperturbed stratification profile is restored and the fine structure is reversible. Correction (28) is proportional to the square of the maximum wave amplitude and is a wave-generated irreversible fine structure.

Calculation results. We will calculate the vertical fine structure generated by the internal wave for 14-minute internal waves of the lowest mode observed at the entrance to the Strait of Gibraltar from the Mediterranean Sea [33]. The amplitude of these waves was $16 \mathrm{~m}$. The vertical profiles of the Brunt Väisälä frequency and the flow velocity are shown in Fig. 1.
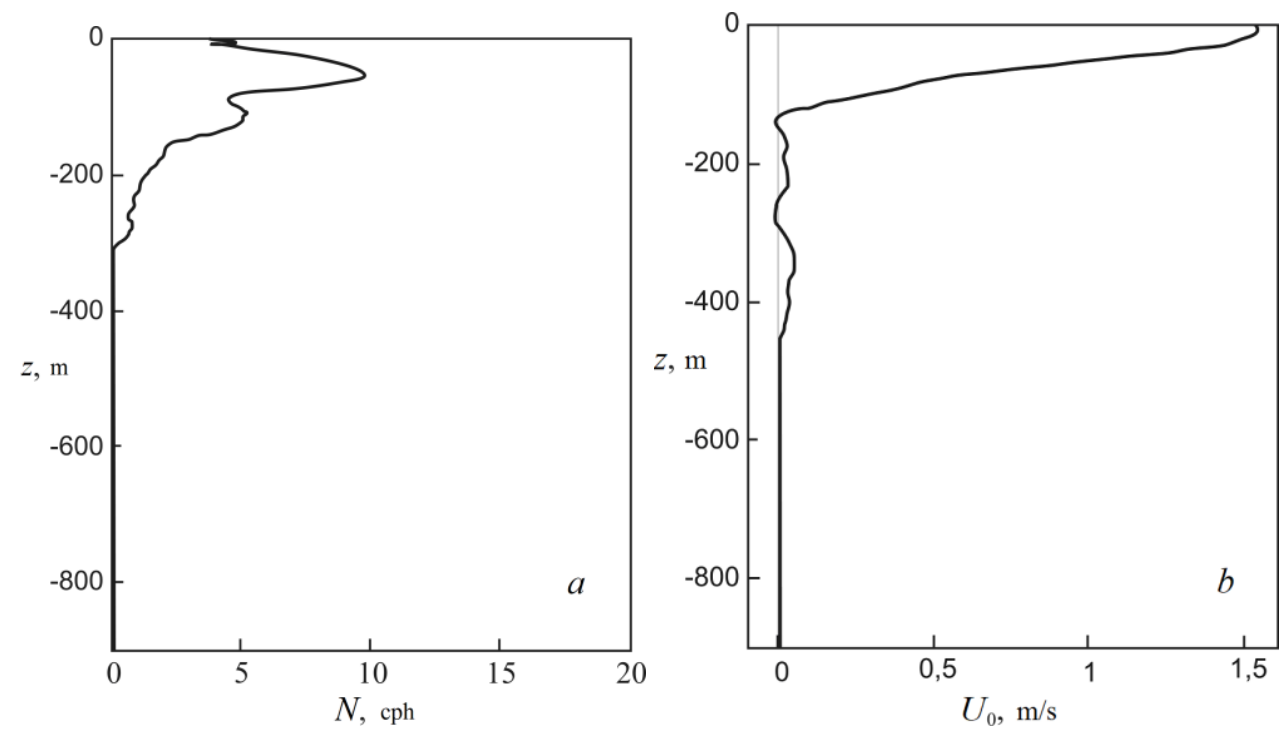

F i g. 1. Vertical profiles of: the Brunt - Väisälä frequency $(a)$; the current velocity $(b)$ 
The boundary value problem (16), (18) is solved numerically by the implicit third-order Adams scheme of accuracy. The dispersion curves of the first two modes are shown in Fig. 2, $a$, with no regard to the flow - in Fig. 2, b. In the lowfrequency region, in the vicinity of the inertial frequency, a significant difference in the behavior of the dispersion curves is observed. When the flow is taken into account, the dispersion curves are cut off in the low-frequency region (Fig. 2, a) due to the effect of critical layers, where the wave frequency with the Doppler shift is equal to inertial one. The minimum frequency for the first mode is $1,326 \cdot 10^{-4} \mathrm{rad} / \mathrm{s}$, for the second mode $-4,363 \cdot 10^{-4} \mathrm{rad} / \mathrm{s}$. For comparison, we indicate that the inertial frequency is equal to $8,582 \cdot 10^{-5} \mathrm{rad} / \mathrm{s}$. In the absence of a flow (Fig. 2, $b$ ), no cutoff occurs in the low-frequency region and the dispersion curves at small wave numbers smoothly approach the inertial frequency. The wave number of 14-minute internal waves of the first mode is $3.76 \cdot 10^{-3} \mathrm{rad} / \mathrm{m}$. We find the normalizing factor $A_{1}$ by the known value of the maximum amplitude of vertical displacements. In order to express the vertical displacement $\zeta$ we use the relation $d \zeta / d t=w$ :

$$
\zeta=\frac{i w_{0}}{\Omega_{0}} A_{1} \exp \left(i k x-i \omega_{0} t\right)+c . c .
$$

This implies

$$
A_{1}=\frac{\max \zeta}{2 \max \left|w_{0} / \Omega_{0}\right|} .
$$
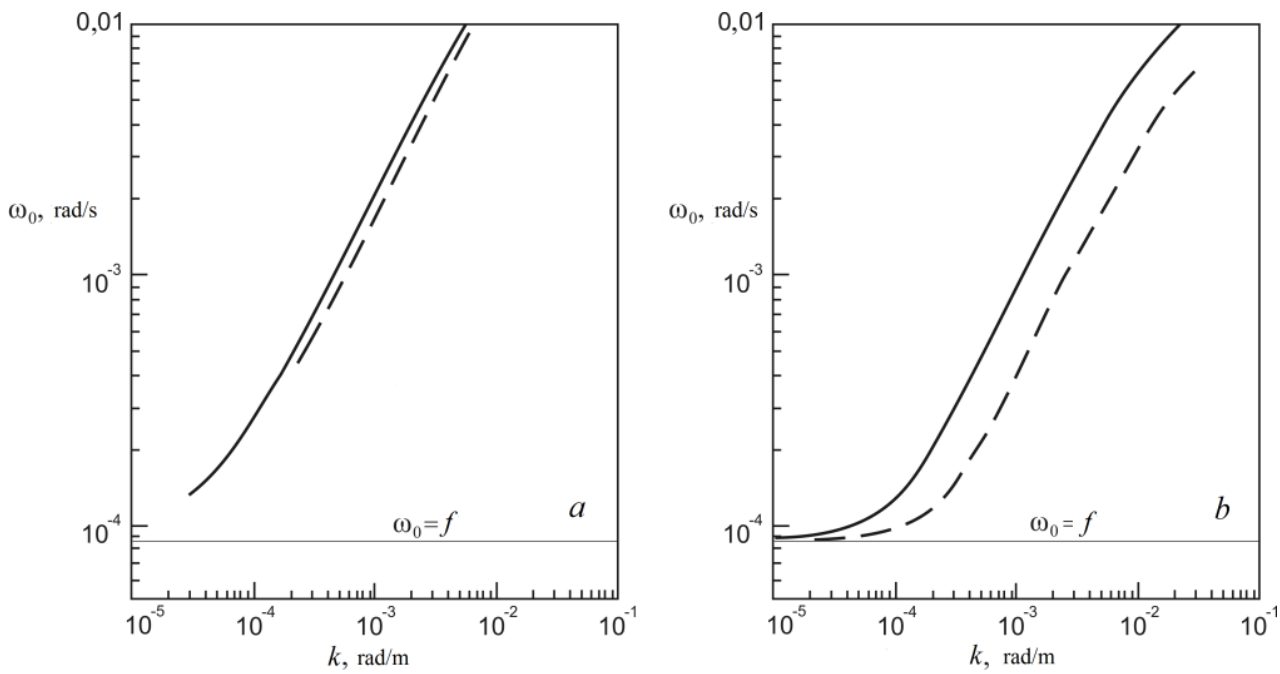

F i g. 2. Dispersion curves of the first two modes of internal waves: $a$ - at presence of the current; $b-$ when the current is absent (the first mode - solid line, the second one - hatch line) 
A typical value of turbulent diffusion coefficient $M$ at the considered scales ${ }^{4}$ is $1 \mathrm{~m}^{2} / \mathrm{s}$. Semi-homogeneous boundary value problem (20), (21) is solved numerically according to the implicit Adams scheme of the third order of accuracy at $K=2 M$. A unique solution that is orthogonal to the nontrivial solution $w_{0}$ of the corresponding homogeneous boundary value problem (15), (16) is found. The correction to the frequency $\omega_{1}$ is found from the solvability condition (22) of this boundary value problem and is determined by formula (23). The value $\omega_{1}$ is purely imaginary, therefore $\delta \omega=\omega_{1} / i$ is a decrement of wave attenuation. In Fig. 3, $a$ the dependence of the wave attenuation decrement on frequency is shown. The decrement modulo of the second mode is larger than the one of the first mode. Features of the decrement behavior in the low-frequency region are shown in Fig. 3, $b$. The decrement reduction in the vicinity of the inertial frequency due to the effect of critical layers, where the frequency of the wave with the Doppler shift is inertial, attracts attention. In the absence of a current, the wave attenuation decrement modulo is at least an order of magnitude larger (Fig. 3, c). No cutoff occurs in the low-frequency region (Fig. 3, $d$ ).

Vertical wave fluxes of mass (24) for 14-minute internal waves of the first mode both in the presence of a current and in its absence are shown in Fig. 4. In the presence of a current, the wave flux of mass is smaller. In Fig. 5 the wave (in the presence of a current) and turbulent vertical mass fluxes are compared. The turbulent flux of mass is determined by the formula $\overline{\rho^{\prime} w^{\prime}}=-M_{z} \frac{d \rho_{0}}{d z}$, the coefficient of vertical turbulent exchange is estimated by the formula $M_{z} \cong 0,93 \cdot 10^{-4} N_{c}^{-1} \mathrm{~m}^{2} / \mathrm{s}, N_{c}$ corresponds to the Brent - Väisäl frequency in cph [34].

The wave flux of the mass exceeds in modulus the turbulent one. The vertical profile of mean density $\rho_{0}$ is represented in Fig. 6, $a$. A non-oscillating at the wave time scale correction to density $\overline{\Delta \rho}(28)$ both in the presence of a current and in its absence, is shown in Fig. 6, $b$. In Fig. 6, $c$, the same dependences are presented in the pycnocline. A non-oscillating at the wave time scale correction to density in the upper 40-meter layer is larger in magnitude in the presence of a current. Inversions in the vertical density distribution do not occur. The characteristic scale of vertical fine structure $(10-20 \mathrm{~m})$ generated by the wave corresponds to the actually observed one.

\footnotetext{
${ }^{4}$ Bowden, K., 1983. Physical Oceanography of Coastal Waters. Somerset, New Jersey: Wiley, 1983).
} 

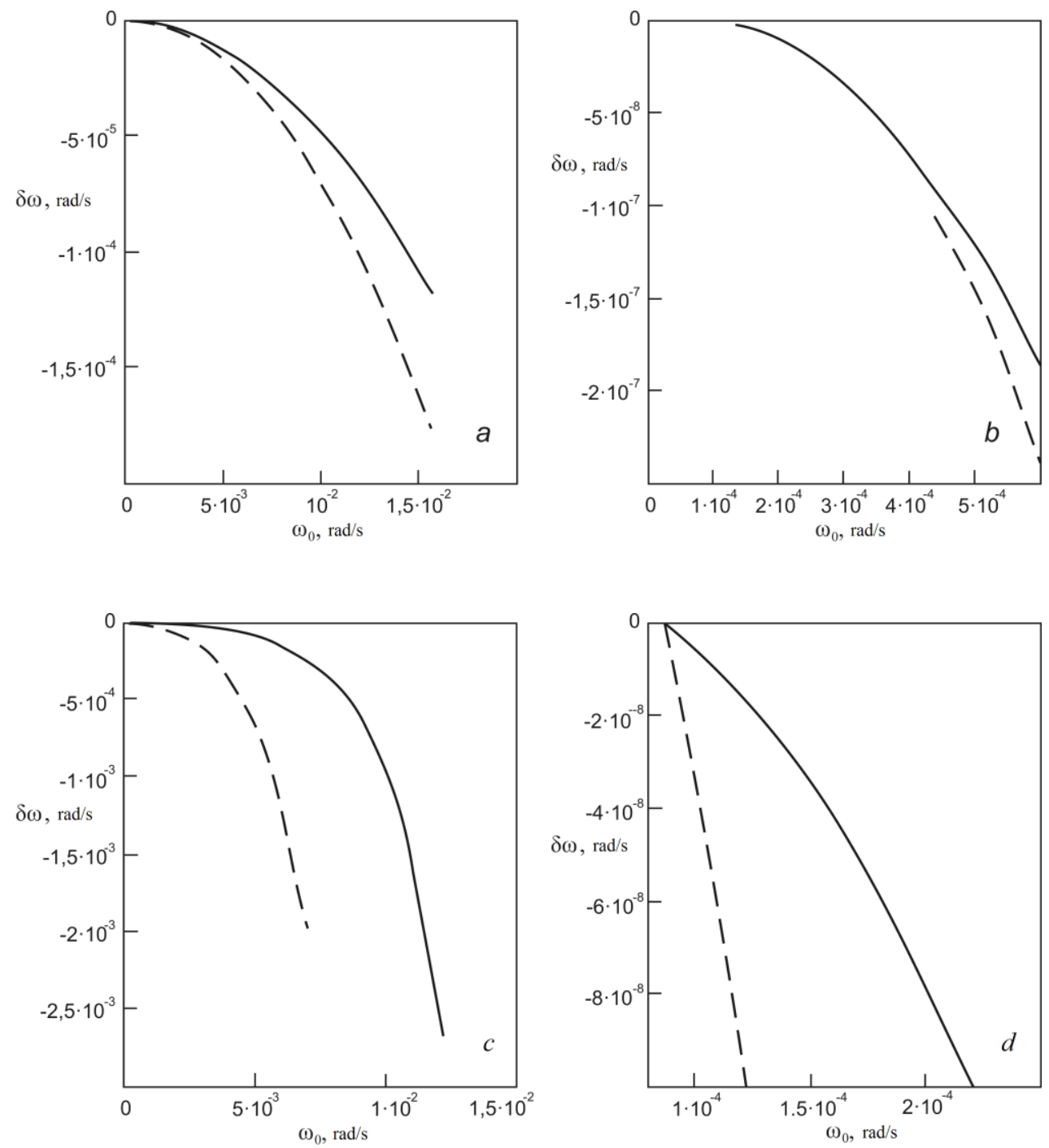

F i g. 3. Dependence of the attenuation decrement on the wave frequency $(a)$; the same - in the lowfrequency area $(b)$; the same dependencies - when the current is absent $(c, d)$ 


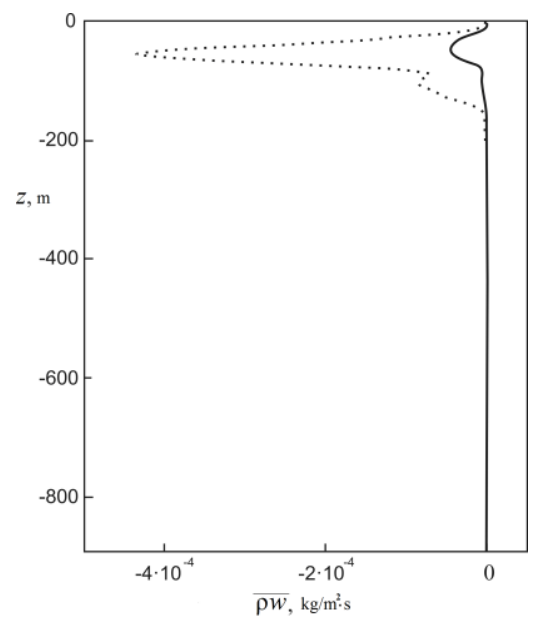

F i g. 4. Profiles of the mass vertical wave flux at presence of the current (solid line) and when the current is absent (dotted line)
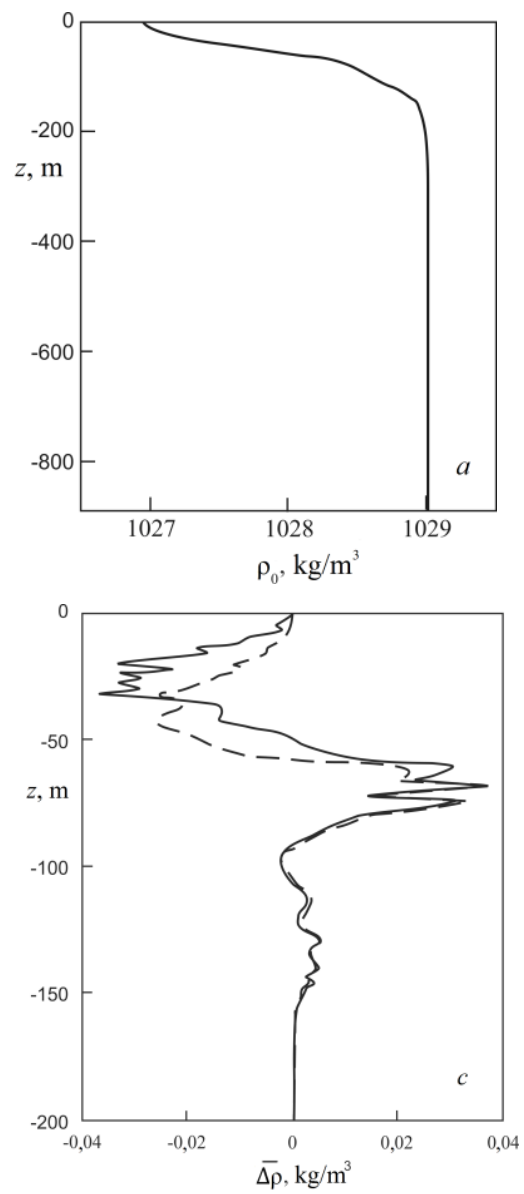

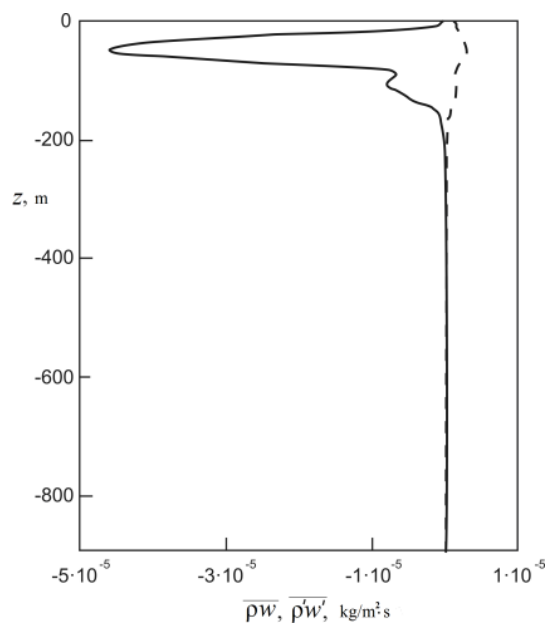

F i g. 5. Profiles of the wave (solid line) and turbulent (hatch line) vertical flux of the mass

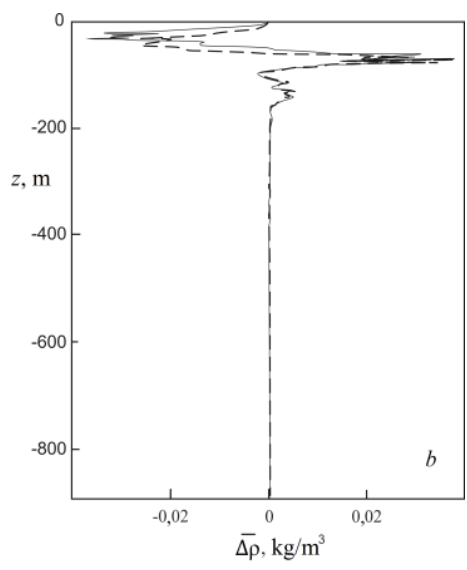

F i g. 6. Vertical profiles: $a$ - of average density; $b$ - of not oscillating correction of density at presence of the current (solid line) and when the current is absent (dotted line); $c$ - the same - in the upper $200 \mathrm{~m}$ layer 


\section{Conclusions}

1. The vertical wave flux of mass with regard to horizontal turbulent viscosity and diffusion is nonzero and exceeds the turbulent one in absolute value.

2. The indicated vertical wave flux of mass leads to non-oscillating on the wave time scale correction to the density - the wave-generated fine structure. This fine structure is irreversible.

3. Dispersion curves of internal waves are cut off in the low-frequency area, which is caused by the effect of critical layers where the wave frequency with the Doppler shift is equal to inertial one.

\section{REFERENCES}

1. Dykman, V.Z., 2016. Technical Tools for Studying Structure and Dynamics of Water Masses. Physical Oceanography, (6), pp. 49-62. doi:10.22449/1573-160X2016-6-43-55

2. Sabinin, K.D. and Serebryanyi, A.N., 2012. Results of Using Acoustic Doppler Current Profilers for Studying the Spatial Structure of the Marine Environment. Acoustical Physics, 58(5), pp. 586-595. https://doi.org/10.1134/S106377101203013X

3. Paka, V.T., Rudels, B., Quadfasel, D. and Zhurbas, V.M., 2010. Measurements of Turbulence in the Zone of Strong Bottom Currents in the Strait of Denmark. Doklady Earth Sciences, 432(1), pp. 613-617. https://doi.org/10.1134/S1028334X10050144

4. Samodurov, A.S., Chukharev, A.M., Zubov, A.G. and Pavlenko, O.I., 2015. Structure-Formation and Vertical Turbulent Exchange in the Coastal Area of the Sevastopol Region. Physical Oceanography, (6), pp. 3-16. doi:10.22449/1573160X-2015-6-3-14

5. Keller, K.H. and Van Atta, C.W., 2000. An Experimental Investigation of the Vertical Temperature Structure of Homogeneous Stratified Shear Turbulence. Journal of Fluid Mechanics, 425, pp. 1-29. https://doi.org/10.1017/S0022112000002111

6. Van Haren, H., Gostiaux, L., Morozov, E. and Tarakanov, R., 2014. Extremely Long Kelvin-Helmholtz Billow Trains in the Romanche Fracture Zone. Geophysical Research Letters, 41(23), pp. 8445-8451. https://doi.org/10.1002/2014GL062421

7. Wunsch, C. and Ferrari, R., 2004. Vertical Mixing, Energy, and the General Circulation of the Oceans. Annual Review of Fluid Mechanics, 36, pp. 281-314. https://doi.org/10.1146/annurev.fluid.36.050802.122121

8. Holford, J.M. and Linden, P.F., 1999. Turbulent Mixing in a Stratified Fluid. Dynamics of Atmospheres and Oceans, 30(2-4), pp. 173-198. https://doi.org/10.1016/S0377-0265(99)00025-1

9. Shroyer, E.L., Moum, J.N. and Nash, J.D., 2010. Energy Transformations and Dissipation of Nonlinear Internal Waves over New Jersey's Continental Shelf. 
Nonlinear Processes in Geophysic, 17(4), pp. 345-360. https://doi.org/10.5194/npg17-345-2010

10. Ostrovskii, A.G. and Zatsepin, A.G., 2016. Intense Ventilation of the Black Sea Pycnocline due to Vertical Turbulent Exchange in the Rim Current Area. Deep Sea Research Part I: Oceanographic Research Papers, 116, pp. 1-13. https://doi.org/10.1016/j.dsr.2016.07.011

11. De Silva, I.P.D., Brandt, A., Montenegro, L.J. and Fernando, H.J.S., 1999. Gradient Richardson Number Measurements in a Stratified Shear Layer. Dynamics of Atmospheres and Oceans, 30(1), pp. 47-63. https://doi.org/10.1016/S03770265(99)00015-9

12. Troy, C.D. and Koseff, J.R., 2005. The Instability and Breaking of Long Internal Waves. Journal of Fluid Mechanics, 543, pp. 107-136. doi:10.1017/S0022112005006798

13. Clark, H.A. and Sutherland, B.R., 2010. Generation, Propagation, and Breaking of an Internal Wave Beam. Physics of Fluids, 22(7), 076601. doi:10.1063/1.3455432

14. Podymov, O.I., Zatsepin, A.G. and Ostrovsky, A.G., 2017. Vertical Turbulent Exchange in the Black Sea Pycnocline and Its Relation to Water Dynamics. Oceanology, 57(4), pp. 492-504. https://doi.org/10.1134/S0001437017040142

15. Vasil'ev, O.F., Voropaeva, O.F. and Kurbatskii, A.F., 2011. Turbulent Mixing in Stably Stratified Currents of the Environment: The Current State of the Problem (Review). Izvestiya, Atmospheric and Oceanic Physics, 47(3), pp. 265-280. https://doi.org/10.1134/S000143381103011X

16. Samodurov, A.S., Chukharev, A.M., Nosova, A.V. and Globina, L.V., 2013. Internal Waves Intensification in the Shelf Break Region as a Factor of the Vertical Exchange Intensification. Fundamentalnaya i Prikladnaya Gidrofizika, 6(2), pp. 1224 (in Russian).

17. Zhurbas, V.M. and Ozmidov, R.V., 1983. On the Internal Structure of the Fine Stepped Structure of the Main Ocean Thermocline. Oceanology, 23(6), pp. 938-943.

18. Falina, A.S. and Volkov, I.I., 2005. Influence of Double Diffusion on the General Hydrological Structure of the Deep Waters in the Black Sea. Oceanology, 45(1), pp. 16-25.

19. Pogrebnoi, A.E. and Samodurov, A.S., 2014. Evolution of Mixed Layers in a Stratified Region of the Black Sea Anticyclonic Eddy. Izvestiya, Atmospheric and Oceanic Physics, 50(6), pp. 621-629. doi:10.7868/S0002351514060121

20. Romanenkov, D.A., Zimin, A.V., Rodionov, A.A., Atazhanova, O.A. and Kozlov, I.E., 2016. Variability of Fronts and Features of Mesoscale Water Dynamics in the White Sea. Fundamentalnaya $i$ Prikladnaya Gidrofizika, 9(1), pp. 59-72 (in Russian).

21. Maurer, B.D., Bolster, D.T. and Linden, P.F., 2010. Intrusive Gravity Currents between Two Stably Stratified Fluids. Journal of Fluid Mechanics, 647, pp. 53-69. doi:10.1017/S0022112009993752

22. Borisenko, Yu.D., Voronovich, A.G., Leonov, A.I. and Miropolsky, Yu.Z., 1976. On the Theory of Nonstationary Weak Nonlinear Internal Waves in Stratified Fluid. Izvestiya AN SSSR, Fizika Atmosfery i Okeana, 12(3), pp. 293-301 (in Russian). 
23. Voronovich, A.G., Leonov, A.I. and Miropolsky, Yu.Z., 1976. On the Theory of Formation of Fine Structure of Hydrophysical Fields in the Ocean. Okeanologiya, 16(5), pp. 750-759 (in Russian).

24. Le Blon, P.H. and Misek, L.A., 1978. Waves in the Ocean. Amsterdam: Elsevier Scientific Publishing Company. Pt. 2, 365 p.

25. LeBlond, P.H., 1966. On the Damping of Internal Gravity Waves in a Continuously Stratified Ocean. Journal of Fluid Mechanics, 25(1), pp. 121-142. doi:10.1017/S0022112066000089

26. Slepyshev, A.A., 2015. Vertical Fluxes Induced by Weak-Nonlinear Internal Waves in a Baroclinic Flow. Physical Oceanography, (1), pp. 59-72. doi:10.22449/1573160X-2015-1-59-72

27. Slepyshev, A.A., 2016. Vertical Momentum Transfer by Internal Waves when Eddy Viscosity and Diffusion are Taken into Account. Izvestiya, Atmospheric and Oceanic Physics, 52(3), pp. 301-308. doi:10.1134/S0001433816030117

28. Vishik, M.I. and Lyusternik, L.A., 1957. Regular Degeneration and Boundary Layer for Linear Differential Equations with Small Parameter. Uspekhi Matematicheskikh Nauk, 12(5), pp. 3-122 (in Russian).

29. Banks, W.H.H., Drazin, P.G. and Zaturska, M.B., 1976. On the Normal Modes of Parallel Flow of Inviscid Stratified Fluid. Journal of Fluid Mechanics, 75(1), pp. 149-171. doi:10.1017/S0022112076000153

30. Booker, J.B. and Bretherton, F.P., 1967. The Critical Layer for Internal Gravity Waves in a Shear Flow. Journal of Fluid Mechanics, 27(3), pp. 513-539. doi:10.1017/S0022112067000515

31. Caillol, P. and Grimshaw, R.H.J., 2012. Internal Solitary Waves with a Weakly Stratified Critical Layer. Physics of Fluids, 24(5), 056602. doi:10.1063/1.4704815

32. Jones, W.L., 1967. Propagation of Internal Gravity Waves in Fluids with Shear Flow and Rotation. Journal of Fluid Mechanics, 30(3), pp. 439-448. doi:10.1017/S0022112067001521

33. Watson, G., 1994. Internal Waves in a Stratified Shear Flow: The Strait of Gibraltar. Journal of Physical Oceanography, 24(2), pp. 509-517. https://doi.org/10.1175/1520-0485(1994)024<0509:IWIASS>2.0.CO;2

34. Ivanov, V.A., Samodurov, A.S., Chuharev, A.M. and Nosova, A.V., 2008. Intensification of Vertical Turbulent Exchange in the Interface between the Shelf and the Continental Slope in the Black Sea. Reports of the National Academy of Sciences of Ukraine, (6), pp. 108-112 (in Russian).

About the authors:

Aleksandr A. Slepyshev, Leading Research Associate, Marine Hydrophysical Institute of RAS (2 Kapitanskaya St., Sevastopol, 299011, Russian Federation), Dr. Sci. (Phys.-Math.) ResearcherID: V-6948-2017, ORCID ID: 0000-0002-9259-7558, slep55@mail.ru

Anna V. Nosova, Leading Engineer-Researcher, Marine Hydrophysical Institute of RAS (2 Kapitanskaya St., Sevastopol, 299011, Russian Federation) 
Contribution of the co-authors:

Aleksandr A. Slepyshev - the statement of the problem, the theoretical part of the work, including obtaining the basic formulas

Anna V. Nosova - calculations, numerical solution of boundary value problems of the second order according to the implicit Adams scheme of the third order of accuracy, a graphical representation of the results

All the authors have read and approved the final manuscript.

The authors declare that they have no conflict of interest. 